\title{
Pesquisas cooperativas entre universidades e institutos públicos no setor agropecuário brasileiro: um estudo na Embrapa*
}

\author{
Alvaro Augusto Dossa** \\ Andréa Paula Segatto***
}

Sumário: I. Introdução; 2. A tecnologia e a inovação na sociedade; 3. Cooperação interinstitucional para inovação tecnológica; 4. Pesquisa agropecuária brasileira; 5 . Metodologia; 6. Apresentação dos resultados; 7. Considerações finais.

Summary: 1. Introduction; 2. Technology and innovation within the society; 3 . Interinstitutional cooperation for technological innovation; 4. Agricultural research in Brazil; 5. Methodology; 6. The results; 7. Final considerations.

Palavras-chave: Sistema Nacional de Inovação; pesquisa agropecuária brasileira; cooperação universidade-instituto de pesquisa.

KEY WORDS: National Innovation System; Brazilian agricultural research; research institutes-university cooperation.

A presente pesquisa objetivou a caracterização das cooperações entre universidades e institutos de pesquisa (U-IP) no setor agropecuário. O referencial teórico aborda temas como tecnologia e inovação tecnológica, sistema nacional de inovação (SNI), cooperação interinstitucional para inovação tecnológica, transferência de tecnologia, além dos papéis de universidades e institutos de pesquisa dentro do SNI. Também

\footnotetext{
* Artigo recebido em jan. 2010 e aceito em ago. 2010.

** Graduado em administração internacional de negócios e mestre em administração pela Universidade Federal do Paraná (UFPR). Endereço: Rua São Pedro, 575 - Cabral — CEP 80.035-020, Curitiba, PR, Brasil. E-mail: alvaroaugusto@gmail.com.

*** Doutora em administração pela Universidade de São Paulo (USP). Docente do Programa de Pós-Graduação em Administração (PPGADM) da Universidade Federal do Paraná (UFPR). Coordenadora do Grupo de Estudos em Gestão da Inovação Tecnológica (Egit). Endereço: Programa de Pós-Graduação em Administração/UFPR — Av. Pref. Lothário Meissner, 632/2ํandar - CEP 80210-170, Curitiba, PR, Brasil. E-mail: aps@ufpr.br.
} 
aborda os temas de tipos de pesquisa e o contexto da pesquisa agropecuária brasileira. A revisão teórica foi fundamentada na cooperação entre universidades e empresas (U-E), dada a reduzida literatura a respeito da cooperação U-IP. A metodologia utilizada foi a de estudo de caso, de natureza exploratória e qualitativa, utilizando roteiros de entrevista semiestruturados, questionários semiestruturados, bem como análise documental. O caso estudado foi o da Empresa Brasileira de Pesquisa Agropecuária (Embrapa), onde foram coletados dados em três unidades de pesquisa, três universidades que cooperam com essas unidades e três unidades administrativas da Embrapa que apresentam contato com as universidades, totalizando nove entrevistas e questionários. Foi possível dessa forma identificar motivadores, tipos de ligações, barreiras, facilitadores e resultados percebidos nessas cooperações.

The research cooperation between universities and public research institutes in the Brazilian agricultural sector: a case study of Embrapa

This research aims the characterization of the university-research institutes cooperation process in the agriculture. The theoretical referential approaches themes such as technology and technological innovation, national innovation system, cooperation between organizations for technological innovation, technology transfer, and the universities and research institutes roles in the Brazilian national innovation system. It also addresses the types of research and the Brazilian agricultural research context. The theoretical review was based in the universit-industry cooperation, since there is too little literature regarding the research institute-university cooperation. The methodology of case study was used, and the research nature was classified as exploratory and qualitative, and used semi-structured interviews and questionnaires, as well as documental analysis. The case studied was of Embrapa, were the data was collected in three research units, three universities that cooperate with these units, and three administrative units of Embrapa that have contact with universities, in a total of nine interviews and questionnaires. It was possible this way identify motivators, types of links, barriers, facilitators and perceived results in these kind of cooperation's.

\section{Introdução}

A sociedade contemporânea apresenta como sistema econômico dominante um modelo capitalista conhecido por pautar-se na inovação (Schumpeter, 2002) e no conhecimento, sendo chamado de Economia Baseada no Conhecimento, ou ainda Economia Baseada no Aprendizado (Johnson e Lundvall, 2005). Tal perspectiva trabalha com a noção de que conhecimento é um dos principais recursos - senão o principal — para indivíduos e organizações realizarem inovações, tornando-se ou permanecendo competitivos (Courvisanos, 2009; Huang, 2009). 
Kim e Nelson (2005), Jonhson e Lundvall (2005) e Santamaría, BargeGil e Mondrego (2010) complementam essa visão e afirmam que uma das principais forças motoras dos países industrializados tem sido o avanço tecnológico. O exemplo dos Tigres Asiáticos é seminal nesse sentido já que, entre as razões para o rápido desenvolvimento desses países de industrialização recente, encontram-se taxas elevadas de investimento em capital humano e físico, o espírito empreendedor com a assunção de riscos, o aprendizado eficaz e a inovação. Tais países passaram de economias pobres e tecnologicamente defasadas para economias afluentes e modernas (Kim e Nelson, 2005). Para Porter (1990) e Courvisanos (2009), inovar é uma das únicas formas de assegurar a vantagem competitiva. Assim, é fundamental o entendimento de como se dá o processo de geração da inovação.

A relevância do papel da cooperação interorganizacional em prol da inovação é evidenciada nas literaturas científicas. Um dos pontos em comum entre os muitos trabalhos que estudam essas ligações é de que a natureza distinta das entidades participantes influencia suas interações. Somado a isso, trabalhos como os de Quental, Gadelha e Fialho (2001), Quental e Gadelha (2000), Ferreira Jr. e Segatto-Mendes (2009) e Steiner (2005) apontam a existência de diferenças entre universidades e institutos de pesquisa, apesar de serem muitas vezes, nos artigos e trabalhos sobre o tema cooperação, considerados elementos homogêneos entre si.

Portanto, um dos pressupostos básicos do presente estudo é o de que cooperações entre universidades e institutos de pesquisa (U-IP) são diferentes de cooperações entre empresas (E-E), universidades e empresas (U-E) e institutos de pesquisa e empresas (IP-E). Assim, o problema de pesquisa estudado neste artigo se caracteriza pela preocupação com a identificação dos elementos diferenciadores do contexto das cooperações nessas distintas instituições.

Adicionalmente, a seleção dos casos estudados teve por base o setor agropecuário. Primeiramente porque, no Brasil, o agronegócio foi responsável em 2008 por $23,7 \%$ do produto interno bruto (PIB) e foi o elemento que mais cresceu de 2007 para 2008 na economia, além de corresponder, em 2008, a $38,4 \%$ do resultado financeiro exportado nesse mesmo ano (Mapa, 2008). Em segundo lugar, conforme Dossa et al. (2009), o Brasil apresentou nas últimas décadas um crescimento na produção agropecuária com um aumento na produtividade, tendência que deverá ser mantida pelos próximos anos, e parte importante desse aumento de produtividade se deve à pesquisa científica agropecuária.

Assim, a questão de pesquisa que norteou o estudo aqui apresentado foi: como se caracterizam os processos de pesquisa cooperativa entre universi- 
dades e institutos de pesquisa (U-IP), visando o desenvolvimento conjunto de inovações tecnológicas no setor agropecuário?

A resposta a esse problema passou pelo alcance de determinados objetivos, em pesquisas cooperativas U-IP, os quais foram: levantar motivadores percebidos pelos participantes, identificar tipos de ligações das cooperações tecnológica de pesquisa no setor agropecuário, levantar barreiras e facilitadores percebidos e identificar resultados alcançados.

A lacuna existente na literatura sobre as cooperações interorganizacionais U-IP ficou evidenciada no levantamento bibliográfico feito em todas as edições publicadas no período de janeiro de 2002 a agosto de 2010 em revistas científicas de administração. Os periódicos nacionais consultados foram: Revista de Administração Contemporânea (RAC), RAC-Eletrônica, Brazilian Administration Review (BAR), Revista de Administração de Empresas (RAE), RAE Eletrônica, Revista de Administração da USP (RAUSP), Revista de Administração Mackenzie (RAM), Revista de Administração Eletrônica (REAd), Revista de Administração Pública (RAP), Revista Brasileira de Inovação (RBI), Parcerias Estratégicas e Ciência Rural.

Já os periódicos internacionais buscados foram: $R \& D$ Management, Technovation, Journal of High Technology Management Research, Research Policy, Technological Forecasting and Social Change, Journal of Business Research, Journal of Technology Transfer e Research Technology Management. No entanto, não foram encontrados artigos sobre o tema com as especificidades do estudo proposto, ou seja, não foram encontrados estudos que objetivassem caracterizar a cooperação U-IP para inovação tecnológica.

Além desses periódicos, também foi feita uma busca por artigos que trabalhassem exclusivamente a cooperação entre universidades e institutos de pesquisa nos anais dos eventos: Academy of Management 2008; Altec 2003 Balas 2000, 2003, 2004 e 2005; CLADEA 2002 e 2004; Congresso Iberoamericano de CTS + I 2006; E3S 2003; Egepe 2005; Enampad 1997 a 2008; Enegep 2005; Eneo 2004; Epege 2005; Iamot 2005 e 2007; Semead 2005; Simpoi 2005; Simpósio de Gestão da Inovação 1998 a 2008 (evento bianual). Os autores compreendem que estudos sobre a cooperação U-IP podem ter sido publicados em outros meios. Contudo, a relevância dessas fontes para a área e a atualidade dos trabalhos por elas publicados permite supor que a ausência da temática em suas edições indica uma lacuna que essa pesquisa procurou reduzir, aumentando o conhecimento sobre esses específicos processos cooperativos.

Quanto às justificativas práticas da pesquisa, é preciso considerar o contexto econômico em que o estudo foi feito. Os estudos das cooperações inte- 
rinstitucionais para inovação tecnológica apresentam como um dos maiores motivadores a redução nos custos e o aumento da eficiência financeira dos projetos (Rapini e Righi, 2006; Duso, Pennings e Seldeslachts, 2010; Cruz e Segatto, 2009). Em se tratando da cooperação U-E e IP-E, a participação das empresas nesses projetos cooperativos está muito relacionada ao investimento por parte das empresas em projetos com organizações que historicamente apresentam redução em seus recursos para P\&D, no caso, institutos de pesquisa e universidades (Ferreira Jr., 2006; Mendes e Segatto-Mendes, 2006; Chagas e Ichikawa, 2009).

Nesse contexto, é necessária a racionalização e a procura por maior eficiência e eficácia da aplicação dos recursos voltados para projetos de inovação tecnológica. Tendo em vista que essa pesquisa procurou ampliar o entendimento do processo cooperativo U-IP, entende-se que poderá ajudar a construir instrumentos que permitam reduzir desperdícios de recursos nessas pesquisas em parceria (Santamaría, Barge-Gil e Mondrego, 2010) e colaborar no desenvolvimento de ferramental útil a esses trabalhos.

Cabe esclarecer que no presente estudo, a Embrapa é o instituto de pesquisa estudado, pois é um importante elemento do sistema nacional de inovação do Brasil, conforme será melhor detalhado ao longo deste trabalho.

\section{A tecnologia e a inovação na sociedade}

Tecnologia, de acordo com Aunger (2010), é um conceito bastante complexo de ser definido e bastante difuso. Tigre (2006) define tecnologia como conhecimento sobre técnicas, que por sua vez englobam aplicações desse conhecimento em produtos, processos e métodos. Já Reis (2004) define tecnologia como um conjunto de conhecimentos de uma sociedade, mas que se relaciona com as artes industriais. Fica claro, portanto, que apesar de haverem diversos conceitos, tecnologia envolve não apenas conhecimento, mas também sua aplicação.

Tendo em vista a importância da tecnologia no tema inovação, faz-se importante definir inovação tecnológica. Andreassi (2007) define inovação tecnológica de forma bastante semelhante à definição de inovação de uma perspectiva schumpeteriana (Schumpeter, 2002), pois considera quando a inovação de produto ou processo é acompanhada pela inserção de novas tecnologias. Em outras palavras, quando a inovação de produto ou processo é acompanhada de conhecimento científico e empírico empregados em algum ramo de atividade (Andreasi, 2007:2). 
Rogers (2003) aponta que a maioria das inovações tecnológicas são criadas pela pesquisa científica, resultando frequentemente da interação entre problemas práticos e método científico. Assim, inovação tecnológica pode ser definida como a inovação que parte do reconhecimento de uma necessidade ou problema bem como aquela que parte da pesquisa científica (Noveli, 2006).

Cohen, Nelson e Walsh (2002) e Balconi, Brusoni e Orsenigo (2010) descrevem a visão predominante sobre inovação até a década de 1970, quando ela era percebida de forma linear, sendo originada na pesquisa básica, indo para a aplicada e então para o desenvolvimento e comercialização. Nessa visão tradicional, a pesquisa pública ocorre no início da cadeia e independente do desenvolvimento tecnológico que, por sua vez, é resultado do depósito comunitário dos resultados de pesquisa.

Para Plonski (1999), o modelo linear de inovação não é o mais adequado, tendo em vista que a sociedade contemporânea apresenta ciência e pesquisa de forma bastante dinâmica, levando em conta que houve um aumento do número de locais potenciais para desenvolvimento de conhecimento, e não apenas as universidades, como por exemplo institutos de pesquisa, laboratórios industriais, consultorias etc. e suas interações. Essa visão é corroborada pelo trabalho de Balconi, Brusoni e Orsenigo (2010), que faz uma compilação de diversas críticas ao modelo linear, e por Noveli (2006), ao afirmarem que a inovação não deve ser percebida como um processo linear, mas como um processo complexo, resultado da interação de diversos agentes e instituições. Esses atores formam uma rede de conexões bastante interdependentes.

O processo inovativo é resultado de um processo de aprendizagem, sendo esse caracterizado pela interação dinâmica entre diversos agentes, cuja reunião das competências leva a avanços nos paradigmas e trajetórias tecnológicas existentes, ou mesmo à conformação de novos paradigmas e trajetórias (Plonski, 1999; Noveli, 2006).

\section{Cooperação interinstitucional para inovação tecnológica}

Em meados da década de 1980, Christopher Freemann utilizou pela primeira vez o conceito de Sistema Nacional de Inovação - SNI (Sharif, 2006; Johnson e Lundvall, 2005), arcabouço teórico posteriormente desenvolvido por outros trabalhos e autores e como Freemann, Nelson e Lundvall (Johnson e Lundvall, 2005). Esse arcabouço apresenta a importância da análise do papel dos diferentes atores econômicos no crescimento nacional e de suas inter-relações. 
No artigo "Promovendo sistemas de inovação como resposta à economia do aprendizado crescentemente globalizada", Johnson e Lundvall (2005), continuando seus estudos no tema, enfatizam a importância da existência de interações fortes entre empresas e instituições diversas, de modo a desenvolver novos conhecimentos em ciência e tecnologia. Dessa forma, organizações governamentais, redes de empresas, institutos de pesquisa, política de ciência e tecnologia, entre outros elementos, dão o suporte necessário para a realização de atividades de P\&D feitos pelo centro desse sistema, as empresas inovadoras (Dantas e Bell, 2009).

Rapini e Rigui (2006), Faria, Lima e Santos (2010), entre outros, ressaltam a importância da interação entre instituições de naturezas diversas como universidades, institutos de pesquisas, empresas etc. em prol do desenvolvimento de inovações. Entre os modelos com mais adeptos que trabalham com tal perspectiva, destaca-se o da Hélice Tríplice (Triple Helix) (Sharif, 2006).

Desenvolvido por Etzkowitz e Leydesdorff (1996, apud Etzkowitz e Leydesdorff, 2000; Park e Leydesdorff, 2010), esse modelo indica que as triplas hélices podem ser vistas em três estágios (Figura 1). A Tripla Hélice 1 é também conhecida como Modelo Estadista de Cooperação U-E, pois nela o governo engloba as universidades e as empresas, esquematizando as cooperações e direcionando as relações a serem estabelecidas. A Tripla Hélice 2 apresenta clara separação institucional entre os vértices e delimitação de cada um, e o governo no vértice superior mantém a função de incentivador de cooperações. A representação é também chamada de modelo laissez-faire. A Tripla Hélice 3 é um modelo em que uma infraestrutura de conhecimento é gerada e as esferas institucionais estão sobrepostas, mostrando que uma pode desempenhar o papel de outra, permitindo surgimento de organizações híbridas (Noveli, 2006).

\section{Figura 1}

Representações dos estágios de desenvolvimento da Tripla Hélice

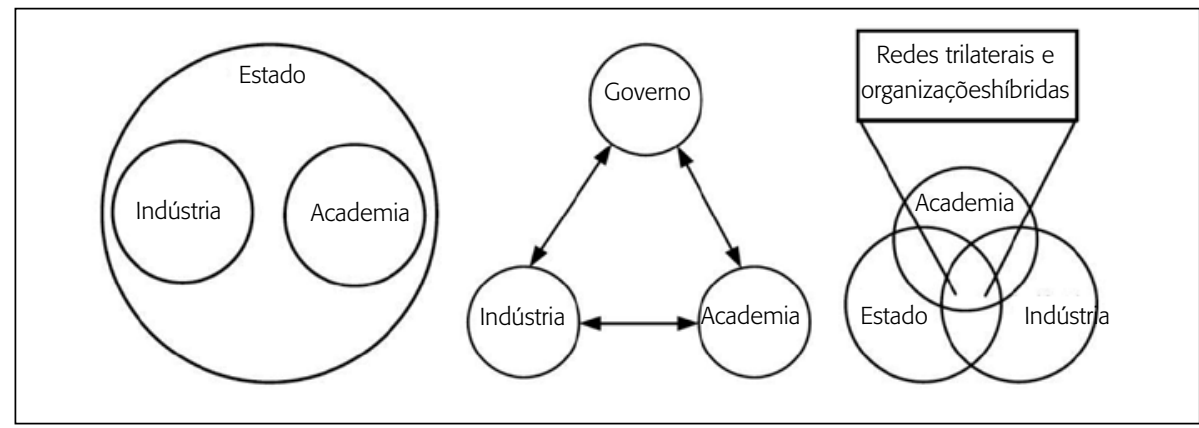

Fonte: adaptado de Etzkowitz e Leydesdorff (2000:111) 
Discorrendo brevemente sobre a questão do papel que as universidades e institutos de pesquisa apresentam em um SNI, Sáez, Marco e Arribas (2002) argumentam que tais instituições são agentes necessários para o desenvolvimento tecnológico de uma nação, bem como nas atividades ligadas ao processo inovativo. Essas instituições devem ser vistas também como fundamentais para a produção, difusão, transformação e desenvolvimento do conhecimento científico e tecnológico.

O modelo da tripla hélice de Etzkowitz coloca as universidades como atores econômicos e sociais na sociedade contemporânea, especialmente conhecida como sociedade do conhecimento. Não apenas isso, mas também considera que as universidades aumentarão sua dependência da economia de conhecimento, além de acrescentar um papel de identificação e guia de ações futuras na produção do conhecimento e suas implicações para a sociedade. Etzkowitz considera que o contexto presente favorece o desenvolvimento de um modelo de universidade empreendedora, buscando atender às alterações e demandas dos sistemas nacionais e regionais de inovação. Dessa forma, os governos passam a considerar que as universidades são agentes importantes para o desenvolvimento econômico e social, já que são atores chave no processo de criação e difusão de inovações e tecnologias (Just e Huffman, 2009; Prado, Porto e Mecenas, 2002).

O papel dos institutos de pesquisa (IPs) em cada país depende das características do SNI em que estão inseridos. Os IPs tendem a dar suporte às atividades de inovação, bem como suporte às empresas com pesquisas direta e indiretamente ligadas às atividades da firma, além de serviços de desenvolvimento tecnológico (Ferreira Jr., 2006).

Arnold et al. (1998) afirmam que o melhor modelo para determinado IP, em um dado período de tempo, depende das necessidades de seus usuários e do próprio instituto, bem como da expertise própria da organização, do estado de desenvolvimento do SNI no qual ele desempenha um papel e da exclusividade de sua contribuição para o funcionamento desse mesmo sistema. Além disso, Dossa (2010) e Porto et al. (2006) lembram que o papel de um IP também varia conforme se privilegia mais ou menos o papel do mercado na indução da inovação e do crescimento econômico.

Os termos aliança, parceria, cooperação, colaboração e interação são utilizados por autores como Nummela (2003), sem o estabelecimento de distinções. Tratamento também adotado para os termos nesse artigo. Considerando essa ausência de distinção, Porto (2004) define cooperação como o resultado da ação conjunta de diferentes atores - a empresa, a universidade e o governo, que desenvolvem parcerias, envolvendo simultaneamente todos 
os atores ou pelo menos dois representantes deles. Uma das definições mais presentes na literatura brasileira sobre cooperação é a de Plonski (1992:VIII apud Segatto-Mendes e Sbragia, 2002:2), que a define como "modelo interinstitucional entre organizações de natureza fundamentalmente distinta, que podem ter finalidades diferentes e adotar formatos bastante diversos", sendo também bastante abrangente, pois "(...) inclui-se neste conceito desde interações tênues e pouco comprometedoras, como o oferecimento de estágios profissionalizantes, até vinculações intensas e extensas, como os grandes programas de pesquisa cooperativa (...)".

É possível afirmar que a inovação tecnológica é hoje um produto de redes de informação e cooperação, em que processos coletivos geram tal inovação. Portanto, necessita de ambientes e contextos apropriados, sendo mais soci-econômica que no passado (Souza, 2010; Suurs e Hekkert, 2009).

Ademais, as organizações públicas de pesquisa (como institutos de pesquisa e universidades públicas) passaram nos últimos anos a procurar desenvolver políticas explícitas em relação à interação interinstitucional com seu ambiente, buscando solucionar os desafios apresentados e encontrar novos parceiros. Entre as estratégias utilizadas destaca-se o aumento das cooperações intra e interinstitucionais, por meio da formação de redes interativas (Gouvea e Kassicieh, 2005; Chagas e Ichikawa, 2009; Dantas e Bell, 2009; Park e Leydesdorff, 2010).

\subsection{0 processo cooperativo interinstitucional}

O processo cooperativo, segundo Segatto-Mendes e Sbragia (2002), se inicia quando o interesse de trabalhar conjuntamente surge nas instituições, de forma a permitir que haja primeiros contratos nesse sentido. O estudo desse interesse, ou seja, o entendimento das motivações para cooperação é importante pela complementaridade que traz ao entendimento do processo cooperativo e suas especificidades (Segatto-Mendes e Rocha, 2005). As motivações adotadas no levantamento feito nesse estudo são oriundas da compilação realizada por Dossa $(2010: 44-45,188)$ para o mesmo contexto.

Tendo em vista que a relação de cooperação em P\&D não é tranquila, já que há diferenças estruturais e de objetivo entre as organizações envolvidas, percepções e expectativas diversas (e às vezes contraditórias) sobre a parceria podem ser geradas, o que dificulta o processo cooperativo (Porto, 2004). Dessa forma, o estudo do processo cooperativo precisa levar em conta essas possíveis barreiras ao trabalho em conjunto das organizações. Cabe ressaltar 
que o trabalho de Noveli (2006) aponta a necessidade de não utilizar apenas o termo barreira, por esse ser visto como impedimento, e a necessidade de utilizar o termo "dificultador" para facilitar o entendimento.

São definidas como barreiras ou dificultadores os possíveis fatores que resultem em problemas para a formação das cooperações ou dificuldades para a continuidade dos trabalhos colaborativos, podendo até mesmo terminar a relação (Segatto, 1996; Segatto-Mendes e Sbragia, 2002). Segatto-Mendes e Rocha (2005) complementam a questão ao afirmar que as barreiras à cooperação não dificultam apenas o andamento do processo colaborativo, mas também o surgimento de novos projetos, já que são dificuldades antecipadas para a relação. As barreiras utilizadas nesse estudo são oriundas da compilação realizada por Dossa $(2010: 46-47,190)$ para o mesmo contexto.

Como aponta Andreassi (2007), embora as motivações para a cooperação interinstitucional - notadamente a colaboração entre universidades e empresas - sejam diferentes para as organizações, elas não são totalmente incompatíveis. Essa cooperação, sendo de natureza complexa, apresenta elementos facilitadores que ajudam a transpor as barreiras, agilizando, melhorando ou mesmo simplificando a cooperação (Segatto, 1996; Segatto-Mendes e Sbragia, 2002). Os facilitadores utilizados nesse estudo, são oriundos da compilação feita por Dossa $(2010: 48-49,191)$ para o mesmo contexto.

São três os tipos básicos de ligações interinstitucionais para o P\&D, de acordo com a tipologia proposta por Vedovello (1997): Ligações Informais: relacionadas ao contato com a fonte de informação e conhecimento, especialidade e equipamento disponível nas universidades ou empresas e suas capacidades e necessidades técnicas e científicas; Ligações de Recursos Humanos: relacionadas ao melhoramento, treinamento e recrutamento e/ou alocação de mão de obra qualificada; e finalmente, Ligações Formais: relacionadas com a exploração das informações técnicas e científicas, conhecimento, especialidade e equipamento disponível em universidades e empresas, pressupondo o estabelecimento de contratos formais entre os cooperados (Vedovello, 1997).

Também é fundamental considerar as questões dos resultados possíveis de tais parcerias. Para Noveli (2006), os resultados do processo cooperativo podem ser tangíveis ou intangíveis, caracterizando os resultados intangíveis como aqueles que não possuem uma dimensão física ou factível, como o aprendizado, e tangíveis, aqueles que a possuem, como por exemplo, artigos publicados em revistas ou conferências, dissertações de mestrado ou teses de doutorado, bem como produtos ou processos licenciados ou não, ou paten- 
tes, licenciadas ou não. Os resultados possíveis das cooperações, adotados no levantamento feito nesse estudo, são oriundos da compilação realizada por Dossa $(2010: 52,191)$ para o mesmo contexto.

\section{Pesquisa agropecuária brasileira}

A pesquisa agrícola brasileira só passou a ser centralizada a partir da criação da Embrapa. Essa instituição, diferente das outras organizações latino-americanas, não integrava a pesquisa e a extensão e coordenava a pesquisa nacional e estadual, já que os estados foram estimulados pela Embrapa a criar institutos próprios de pesquisa, em um modelo parecido com o dela. Ademais, a organização promoveu também a participação privada no tema (Piñero e Trigo, 1985 apud Fuck e Bonacelli, 2007; Chagas e Ichikawa, 2009).

O processo de desenvolvimento das universidades que realizam pesquisa agropecuária foi bastante complexo, especialmente pelo fato de que apenas nos anos de 1930 houve a aprovação da legislação necessária para o estabelecimento de universidades (mesmo havendo alguns casos esparsos, como no Paraná e no Amazonas). As décadas de 1960 e 1970 apresentaram também um aumento na capacitação do quadro de profissionais de quatro universidades: UFRGS, UFV, UFC e USP, resultado especialmente de um acordo com o governo dos Estados Unidos, que permitiu a realização de pós-graduação nesse país para várias pessoas (Beintema, Avila e Pardey, 2001).

A atuação da iniciativa privada na pesquisa agropecuária brasileira iniciou-se principalmente a partir de meados da década de 1940, e como atores relevantes, pode-se citar a Cooperativa dos Produtores de Açúcar e Álcool de São Paulo (Copersucar), a Sementes Agroceres, adquirida em 1996 pela Monsanto, que também adquiriu a Cargill e a Braskalb, sendo agente fundamental do setor, especialmente na pesquisa de sementes (Beintema, Avila e Pardey, 2001).

Em 1992 o Mapa instituiu o Sistema Nacional de Pesquisa Agropecuária (SNPA) vigente, formado pela Embrapa (que também coordena o sistema) e suas unidades, pelos institutos de pesquisa agropecuários estaduais e por diversas universidades (Embrapa, 2008).

Segundo Alves (1992 apud Beintema, Avila e Pardey, 2001), há no Brasil diversas universidades que fazem pesquisa agropecuária, com mais de 100 faculdades ou escolas superiores trabalhando na área. A maior parte dessas instituições são federais ou estaduais, não sendo algo surpreendente já que boa parte da pesquisa brasileira é concentrada nas instituições públicas. Rapini e Righi (2006) apontam que, de acordo com o Censo de 2004 do diretório 
de grupos de pesquisa do CNPq, as instituições de ensino superior públicas representavam $73 \%$ dos grupos e as empresas de pesquisa agropecuária, 4\%, as últimas apresentando enorme dominância da Embrapa. A área "Ciências Agrárias" é o segundo maior grupo com instituições que interagem com grupos de pesquisa interinstitucional. Para os autores, essa realidade é explicada pelo grande incentivo que o governo deu à área, visando o aumento da competitividade do setor.

\section{Metodologia}

Esta pesquisa caracterizou-se como de natureza qualitativa. De acordo com Collis e Hussey (2005), ao classificarem o tipo de pesquisa baseando-se em seu processo, ou seja, a maneira pela qual se coletam e analisam os dados, os estudos qualitativos envolvem o exame e a reflexão das percepções para obter um entendimento de atividades sociais e humanas. Ademais, não há a busca e análise estatística de dados quantitativos (Collins e Hussey, 2005). Cooper e Schindler (2003) apontam que pesquisas qualitativas se referem ao significado, à definição, ao modelo que caracteriza alguma coisa, buscando, portanto, "o que" e não "o quanto".

Foi realizado um estudo de caso, e essa escolha se deu pela capacidade de serem considerados mais convincentes as provas e os resultados obtidos nesse tipo de investigação e, de forma geral, proporcionarem maior robustez ao estudo em questão (Yin, 2005). A análise dos casos foi "dentro do caso" (within-case analysis) e "entre casos" (cross-case pattern search).

Quanto ao objetivo, as pesquisas podem ser classificadas em exploratórias, descritivas ou explicativas/explanatórias (Collins e Hussey, 2005; Neuman, 1997). O presente estudo é caracterizado como exploratório, já que as pesquisas exploratórias são aquelas que buscam encontrar padrões, ideias ou hipóteses, onde o objetivo é o ganho de familiaridade com o tema, geralmente em áreas pouco exploradas ou desenvolvidas, de modo a possibilitar estudos mais rigorosos no futuro. Tendo em vista que não foram encontradas pesquisas relativas à cooperação U-IP durante a busca na literatura, classifica-se a presente pesquisa como exploratória.

Em relação ao horizonte de tempo da pesquisa, o presente estudo se enquadra no tipo cross-sectional, ou seja, no tipo transversal, já que a coleta de dados é limitada a um período específico de tempo, portanto, uma "foto" dos objetos estudados naquele ponto fixo no tempo (Neuman, 1997). Quanto ao nível de análise da pesquisa, caracteriza-se como nível organizacional, 
enquanto as unidades de análise são relacionais, pois buscam retirar informações dos institutos públicos de pesquisa do setor agropecuário e das universidades públicas que realizam pesquisa agropecuária em conjunto com esses institutos.

O modelo de pesquisa utilizado nesse estudo se encontra na Figura 2:

Figura 2

Modelo da pesquisa

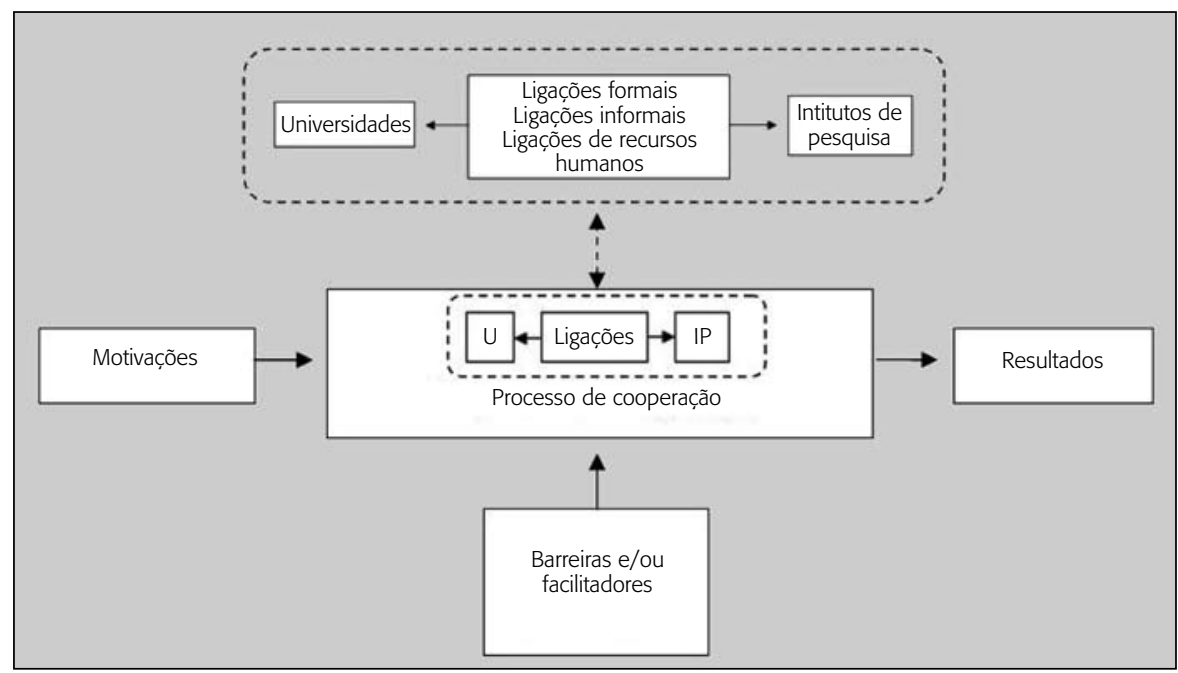

Fonte: adaptado de Noveli (2006).

Foram realizados três grupos de entrevistas, com três pessoas entrevistadas em cada grupo. As unidades administrativas (ou centrais) da Embrapa, o Departamento de Pesquisa e Desenvolvimento (DPD), a Assessoria de Inovação Tecnológica (AIT) e a Assessoria de Relações Nacionais (ARN), por apresentarem relacionamento direto com universidades, foram as unidades que mais puderam agregar para o estudo em relação à visão institucional da Embrapa. Nessas unidades centralizadas escolhidas, os respondentes foram os chefes das unidades ou pessoas indicadas por esses para participar da pesquisa.

As unidades de pesquisa da Embrapa (ou unidades descentralizadas) são divididas em três tipos: Pesquisa Básica, de Produto e Ecorregional. Foi selecionada a unidade, dentro de seu grupo, que mais apresentou publicações em revistas científicas. Dessa forma, foram selecionados: De pesquisa básica, o Centro de Recursos Genéticos e Biotecnologia (Cenargen) em Brasília (DF). De pesquisa de produtos, o Centro Nacional de Pesquisa em Mandioca e Fruti- 
cultura Tropical (CNPMF) em Cruz das Almas (BA). De pesquisa Ecorregional, o Centro de Pesquisa em Clima Temperado (CPACT), em Pelotas (RS).

Nas unidades descentralizadas, foram entrevistados os pesquisadores que mais publicaram artigos de revistas científicas dentro de suas unidades. Foi requisitado aos pesquisadores que apontassem, dentro das universidades que cooperam, quem seria o pesquisador mais indicado para participar dessa pesquisa. Dessa forma, o pesquisador do Cenargen indicou uma colaboradora sua da Unesp-Botucatu, o pesquisador do CNPMF indicou uma professora e pesquisadora da Universidade Federal do Recôncavo Baiano (UFRB) e a pesquisadora do CPACT indicou um professor e pesquisador da Universidade Federal de Pelotas (Ufpel).

Foram realizadas entrevistas semiestruturadas, questionários semiestruturados e pesquisas documentais com todos os respondentes. As categorias de análise teoricamente vinculadas à seção "o processo cooperativo interintitucional", ou seja, motivadores, tipos de ligações, barreiras, facilitadores e resultados, foram apresentados aos entrevistados por meio de tabelas, nas quais cada respondente deveria informar qual seu nível de concordância com cada uma dessas categorias para cada uma das organizações. Assim, cada entrevistado respondeu duas vezes cada categoria, uma vez para sua instituição e uma para a instituição parceira. Ou seja, os entrevistados da Embrapa responderam a partir da visão de sua própria entidade e também expressando sua visão da postura da universidade, e vice-versa.

O consenso entre os grupos a respeito dos elementos perguntados era considerado como obtido desde que ao menos dois integrantes de cada grupo, ou seja, $66,67 \%$ do total, houvessem concordado com a categoria de análise.

\section{Apresentação dos resultados}

\section{Motivadores}

Os questionários demonstraram que os entrevistados apontavam como motivadores consensualmente aceitos nos dois grupos:

- Acesso a recursos materiais, conhecimentos especializados e ativos complementares que reduzem riscos, custos, prazos e incertezas do ambiente inovativo, gerando economias de escopo em P\&D e facilitando os projetos de pesquisa conjunta;

- Resolução de problemas localizados por meio de suporte técnico de excelência; 
- Possibilidade de incorporação de ensino e pesquisa e aquisição de conhecimentos complementares aos seus por meio de acesso a redes de conhecimento novos e internacionais, de troca de experiência e da interação com pesquisadores de diversas instituições;

v Existência de cooperações interinstitucionais anteriores com resultados satisfatórios e bom relacionamento interpessoal;

- Melhoria da imagem pública da instituição ante a sociedade, demonstrando o seu potencial para geração de ciência e tecnologia junto a parceiros, difundindo assim o conhecimento e realizando sua função social;

v Necessidade de atualização tecnológica para manutenção de competitividade.

No entanto, os questionários demonstraram também que nem todos os motivadores referenciados na teoria apresentada, como os do parágrafo anterior, foram apontados pelos participantes da pesquisa. Isto é, não foram identificados de forma consensual pelos respondentes da pesquisa:

v Necessidade de maiores fontes de financiamento para pesquisa;

v Carência de atividade de P\&D na instituição e/ou maiores conhecimentos referentes à pesquisa básica;

- Maior contato com as necessidades de aplicabilidade do conhecimento por parte do mercado/indústria/sociedade;

v Identificação de alunos para recrutamento futuro;

- Possibilidade de acesso a empregos para graduados; o prestígio a ser obtido pelo pesquisador;

- Possibilidade de criação de empresa por pesquisadores participantes da cooperação.

Tais elementos demonstram a existência, nessa categoria, de diferentes leituras dos motivadores em cooperações U-IP, para as interrelações de modo geral, como encontrado na literatura, o que pode ser fruto de diferentes percepções de relevância dos itens da categoria de análise como também de sua inexistência para o contexto específico em estudo.

Do mesmo modo, percebem-se também distinções entre os dois grupos, como no caso do motivador "necessidade de maiores fontes de financiamento para pesquisa", que é entendido como um motivador para as universidades, mas não para a Embrapa. 


\section{Tipos de ligações}

Os resultados obtidos quanto às ligações informais, de recursos humanos e formais, que foram concordadas por todos os grupos de entrevistados foram:

v Contatos informais com pesquisadores/empresários; envolvimento de estudantes em projetos;

v Acesso à pesquisa de departamento científico;

- Consultoria desenvolvida por pesquisadores e consultores;

v Participação em seminários e conferências;

v Análise e testes (ensaios técnicos);

- Acessos a equipamentos da universidade e/ou dos institutos de pesquisa e/ou das empresas;

- Respostas técnicas (diagnósticos de problemas em termos de processo produtivo);

- Participação em programas específicos (educacionais e de treinamento);

v Estabelecimento de pesquisa conjunta;

v Outras ligações informais.

Os tipos de ligação U-E que não puderam ser identificados de forma consensual na cooperação entre a Embrapa e a universidade, de acordo com a tipologia de Vedovello (1997), foram:

v Acesso à literatura especializada;

v Recrutamento de recém-graduados;

- Recrutamento de cientistas e engenheiros mais experientes;

v Programas de treinamento formalmente organizados para atender às necessidades de recursos humanos;

- Serviços de atualização de acervo;

- Estabelecimento de contratos de pesquisa;

v Outras ligações formais ou de recursos humanos.

Entre as ligações identificadas na tipologia de Vedovello (1997), algumas merecem destaque no presente estudo: acesso à literatura especializada, 
recrutamento de cientistas e engenheiros mais experientes e programas de treinamento formalmente organizados para atender as necessidades de recursos humanos, que foram consideradas ligações existentes para a universidade de forma consensual entre os grupos, mas não para a Embrapa. As duas primeiras podem ser indicativos da especialização da Embrapa em conduzir pesquisa agropecuária, diferente da universidade, que apresenta trabalhos em muitas áreas do conhecimento; já a outra ligação pode ser explicada pela natureza das instituições, em que o papel de formação de recursos humanos não é parte das atividades fundamentais da Embrapa, mas é um dos pilares das universidades.

A entrevista semiestruturada apontou que as cooperações universidade-Embrapa são rápidas e não burocráticas e, para a formalização do acordo, quando necessário, o fator humano é fundamental para agilizar o processo.

\section{Barreiras}

O objetivo específico "levantar as barreiras percebidas pelos participantes das pesquisas cooperativas entre universidades e institutos de pesquisa" não conduziu a nenhuma das barreiras utilizadas nos questionários e levantadas na literatura (Dossa, 2010) sobre as cooperações U-E, isto é, não houve concordância com nenhuma barreira pelos três grupos de respondentes. No entanto, as entrevistas semiestruturadas indicaram que a existência de indivíduos dentro das organizações que, por questões pessoais — por exemplo, não fazer parte da pesquisa ou da interação - dificultam ou mesmo não permitem que outros pesquisadores utilizem seus equipamentos e materiais, é uma barreira para cooperações.

Contudo, se considerada apenas barreira para as universidades nas cooperações U-IP, percebeu-se que a "ausência de maior contato do meio acadêmico com a atividade da indústria e com as necessidades do mercado" é percebida consensualmente pelos grupos como um dos dificultadores existentes. Identifica-se aí que é persistente a visão da universidade como organização distante da realidade do mercado na condução das pesquisas, e que isso é uma barreira para a cooperação não apenas com empresas privadas, mas com a Embrapa também.

\section{Facilitadores}


Os facilitadores que, assim como os motivadores e as ligações, apresentaram concordância de pelo menos dois membros de cada um dos grupos - unidades centrais, descentralizadas e pesquisadores da universidade — para ambas as instituições, foram:

- Existência de redes de interação estáveis a fim de facilitar a harmonização de interesses entre os diferentes atores do desenvolvimento tecnológico;

- Boa disponibilidade de informações sobre o parceiro para melhorar compreensão das competências, modo de atuação e especificidades da natureza distinta (como o público e o privado, o científico e o empresarial) das instituições;

v Divulgação de resultados e competências em pesquisa;

v Postura cooperativa do pesquisador;

- Utilização de estruturas que permitam a qualificação, formação e experiência das equipes envolvidas de forma semelhante, como a participação de grupos em programação de pós-graduação e pesquisa de um dos parceiros ou transferência temporária de parte da equipe entre os participantes para diminuir diferenças de linguagem, cultura e experiência técnica, aproximando o nível de conhecimento técnico;

v Existência e efetiva atuação de uma estrutura para gestão das cooperações que explicite a visão institucional dessas interações, o comprometimento das instituições, os objetivos claros e compartilhados, a busca pela boa comunicação, pela confiança mútua e por boas relações interpessoais por parte de instituições parceiras e informações preliminares sobre custos e potencial de mercado;

- Atuação governamental: políticas públicas de incentivo à cooperação.

É interessante destacar que o grupo de categorias de análise do modelo de pesquisa que apresentou maior consenso entre todos os entrevistados, para ambos os tipos de instituições pesquisadas, foi o dos facilitadores, pois todas as opções foram concordadas.

\section{Resultados percebidos}

Da mesma forma que os itens anteriores - motivadores, tipos de ligação e facilitadores -, os resultados percebidos consensualmente foram: 
- Desenvolvimento de novas técnicas e instrumentos e de produtos, processos ou patentes, licenciados ou não;

- Aprendizado incremental: maior capacidade em resolver problemas, melhor identificação das demandas, maior acúmulo de experiência de outras instituições e outros;

v Otimização dos recursos com redução de riscos e economias de tempo;

- Sugestões de novas ideias e acúmulo de experiência na complementação de projetos existentes;

v Realização de novos projetos em conjunto no futuro;

v Melhoria na viabilidade das organizações em longo prazo;

v Maior permeabilidade institucional com elevação das interações entre técnicos e pesquisadores da instituição.

Já os resultados levantados na literatura (Dossa, 2010), que não foram concordados consensualmente pelos entrevistados, foram:

v Aumento de competitividade;

- Publicação de artigos em revistas ou congressos e/ou elaboração de dissertações de mestrado ou teses de doutorado;

v Legitimação da atividade institucional.

A respeito dos resultados gerados pela instituição de cada um dos participantes da pesquisa, de acordo com as entrevistas, foi apontado que a Embrapa transfere seus resultados para a sociedade por meio da geração de tecnologias, metodologias e produtos novos, livros, artigos científicos, relatórios de pesquisa e orientação de dissertações e teses. Já as universidades apontaram que fazem essa transferência por meio da formação de recursos humanos, publicações de livros e artigos científicos, relatórios técnicos e também orientações de dissertações e teses.

Apesar de apresentarem resultados parecidos ou mesmo iguais, a ênfase que se dá a cada tipo de resultado é diferente. Todos os entrevistados afirmaram que são esperadas das universidades a formação de pessoal qualificado e a publicação de artigos científicos, enquanto da Embrapa se esperam a resolução de problemas empíricos da agropecuária e a geração de pesquisas aplicadas. 


\section{Outros elementos identificados}

A realização da pesquisa permitiu a identificação de elementos além daqueles delimitados pelas categorias de análise, mas derivados de seu levantamento. Um dos apontamentos possíveis foi a identificação de que as respostas semelhantes entre as unidades, especialmente as centrais, sobre algumas das questões feitas ao longo da pesquisa mostrou uma convergência que a análise detalhada das repostas apontou ser apenas em aspectos mais gerais do tópico abordado. Isso indica que apesar de serem unidades de atuação semelhantes, sua visão da cooperação não é homogênea, o que retrata a ausência de uma identidade organizacional a respeito do tema.

Os dados acerca da fase inicial das cooperações permitiram identificar outros consensos entre os três grupos analisados: as pesquisas cooperativas são tanto básicas quanto aplicadas e dependem do momento da pesquisa e do grupo envolvido; a escolha de com quem cooperar é uma decisão do líder do projeto e dos pesquisadores envolvidos, e não institucional; questões políticas não são consideradas durante a escolha dos parceiros; e o conhecimento pessoal prévio é importante fator para a seleção de com quem cooperar.

\section{Considerações finais}

O caminho para o desenvolvimento econômico e social de um país passa pela sua capacidade de desenvolver inovações. As capacidades inovativas das organizações de ensino e pesquisa de um país - como universidades e institutos - precisam ser utilizadas ao máximo e o uso de recursos deve ser racional e não permitir desperdícios, seja de tempo, dinheiro, estrutura ou mesmo conhecimento. Entre os campos de estudo mais importantes para a pesquisa e a inovação no país está a agropecuária, dada sua relevância para a estrutura produtiva do Brasil, bem como para as aspirações brasileiras em tornar-se um dos líderes no setor.

Tendo em vista que as cooperações entre governo, academia e setor privado são elementos fundamentais para que os recursos colocados à disposição da inovação sejam bem utilizados, diversos foram os trabalhos que buscaram um melhor entendimento dessas ligações. Contudo, os estudos que procuram aumentar a compreensão e efetividade dessas interações para inovação tecnológica tendem a focar as cooperações entre academia e empresa, geralmente considerando o primeiro como um elemento homogêneo, representando tanto universidades como institutos de pesquisa, e sem fazer distinções entre a visão desses dois tipos de instituições. 
Nesse sentido, a presente pesquisa procurou analisar as interações para P\&D entre IPs e universidades, buscando identificar as possíveis distinções, a partir do estudo de cooperações entre um dos mais importantes institutos de pesquisa do país, a Embrapa, e algumas universidades com que coopera.

Para responder a questão de pesquisa norteadora do trabalho, já apresentada na introdução, foi necessário atingir certos objetivos, os quais todos foram alcançados. Dessa forma, foi possível levantar e/ou identificar quais os motivadores, os tipos de ligações, as barreiras, os facilitadores e os resultados nas pesquisas cooperativas universidade-empresa. Todos esses elementos podem ser encontrados na seção de apresentação dos resultados.

Ao atingir esses objetivos, certos elementos da literatura sobre cooperações interorganizacionais para inovação tecnológica não puderam ser verificados consensualmente quando analisados na perspectiva de cooperações U-IP. Alguns exemplos que podem ser citados: o motivador "identificação de alunos para recrutamento futuro", a ligação "estabelecimento de contratos de pesquisa" e o resultado "aumento de competitividade". Ressalta-se que nenhuma das barreiras levantadas por Dossa (2010) foi concordada por todos os grupos para ambas as instituições. De forma oposta, todos os facilitadores foram identificados pelos entrevistados. Outras contribuições deste estudo foram os outros elementos identificados pela pesquisa, mas não previstos nas categorias de análise. Em especial, a ausência de uma visão homogênea na Embrapa, sobre as cooperações U-IP, e a grande importância do conhecimento prévio entre pesquisadores para as pesquisas cooperativas futuras.

Quanto a possíveis sugestões para as instituições estudadas, recomenda-se o desenvolvimento de ferramentas de formalização de parceria entre universidade e Embrapa, mas que mantenham a flexibilidade e agilidade dos processos. Essa formalização permitirá conhecer e controlar os trabalhos conjuntos informais não apenas no início e término do projeto, mas ao longo dos trabalhos, possibilitando uma melhor avaliação dos pesquisadores.

Algumas pesquisas futuras podem ser propostas a partir do estudo feito. Uma delas é a análise do papel dos Labex da Embrapa como motivadores da interação com universidades, na interação com universidades e unidades de empresas multinacionais que estão no exterior. Outra sugestão de estudo seria uma pesquisa para identificar todas as formas e períodos de interações entre a Embrapa e as universidades, a fim de criar métodos de formalização que sejam flexíveis, rápidos e que não afastem os pesquisadores das instituições por causa da burocracia. O desenvolvimento de uma metodologia de estabelecimento de resultados previstos para cada parte e avaliação desses resultados também 
é necessária, mas ressaltando que deve ser adaptável a cada situação e cada interação entre as instituições.

Adicionalmente, tem-se a questão da localização geográfica, pois esse elemento possui características específicas no contexto das pesquisas estudadas, uma vez que cultivares dependem de certas condições climáticas e de solo. Logo, a localização geográfica como motivador ou não do processo exige um estudo próprio e que aprofunde a compreensão da influência desse elemento dentro das especificidades que apresenta no contexto.

Finalmente, recomenda-se uma pesquisa quantitativa com pesquisadores e pessoal administrativo de institutos de pesquisa para verificar a existência de visões distintas do processo de cooperação com universidades entre esses grupos, como parece acontecer na Embrapa. Isso pode indicar se há ou não a necessidade de trabalhar uma melhor interação entre unidades administrativas e pesquisadores em relação a parcerias interinstitucionais.

\section{Referências}

ANDREASSI, T. Gestão da inovação tecnológica. São Paulo: Thomson, 2007.

ARNOLD, E. et al. Strategic planning in research and technology institutes. $R \& D$ Management, v. 28, n. 2, p. 89-100, 1998.

AUNGER, R. Types of technology. Technological Forecasting and Social Change, v. 77, n. 5, p. 762-782, 2010.

BALCONI, M.; BRUSONI, S.; ORSENIGO, L. Research Policy, v. 39, n. 1, p. 1-13, 2010.

BEINTEMA, N.M.; AVILA, A.F.D.; PARDEY, P.G. P\&D agropecuário no Brasil: política, investimentos e perfil institucional. Instituto Internacional de Pesquisa de Políticas Alimentares, 2001.

CHAGAS, P.B.; ICHIKAWA, E.Y. Redes de C\&T em institutos públicos de pesquisa brasileiros: o caso do Instituto Agronômico do Paraná (Iapar). Revista de Administração Pública, v. 43, n. 1, p. 93-121, 2009.

COHEN, W.M.; NELSON, R.R.; WALSH, J.P. Links and impacts: the influence of public research on industrial R\&D. Management of Science, v. 48, n. 1, p. 1-23, jan. 2002.

COLLIS, J.; HUSSEY, R. Pesquisa em administração: um guia prático para alunos de graduação e pós-graduação. 2. ed. Porto Alegre: Bookman, 2005. 
COOPER, D.R.; SCHINDLER, P.S. Métodos de pesquisa em administração. 7. ed. Porto Alegre: Bookman. 2003.

COURVISANOS, J. Political aspects of innovation. Research Policy, v. 38, n, 7, p. 1.117-1.124, 2009.

CRUZ, E.M.K.; SEGATTO, A.P. Processos de comunicação em cooperação tecnológica universidade-empresa: um estudo de caso em universidades federais do Paraná. Revista de Administração Contemporânea, v. 13, n. 3, p. 430-449, 2009.

DANTAS, E.; BELL, M. Latecomer firms and the emergence and development of knowledge networks: the case of Petrobras in Brazil. Research Policy, v. 38, n. 5, p. 829-844, 2009.

DOSSA, D. el al. Projeções do agronegócio Brasil 2008/2009 a 2018/2019. Assessoria de Gestão Estratégica - Ministério da Agricultura, Pecuária e Abastecimento, fev. 2009.

DOSSA, A.A. A cooperação tecnológica entre universidades e institutos públicos de pesquisa no setor agropecuário: um estudo na Embrapa. Dissertação (Mestrado em Administração) - Setor de Ciências Sociais Aplicadas, Universidade Federal do Paraná, Curitiba, 2010..

DUSO, T.; PENNINGS, E.; SELDESLACHTS, J. Learning dynamics in research alliances: a panel data analysis. Research Policy, v. 39, n. 6, p. 776-789, 2010.

EMPRESA BRASILEIRA DE PESQUISA AGROPECUÁRIA. Programa Nacional de Pesquisa e Desenvolvimento da Agropecuária - Pronapa - 2008: integração pesquisa e extensão como fator de sucesso na moderna agricultura brasileira, v. 34, p. 1-291, 2008.

ETZKOWITZ, H.; LEYDESDORFF, L. The dynamics of innovation: from National Systems and "Mode 2" to a Triple Helix of university-industry-government relations. Research Policy, v. 29, p. 109-123, 2000.

FARIA, P.; LIMA, F.; SANTOS, R. Cooperation in innovation activities: the importance of partners. Research Policy, v. 39, n. 8, p. 1.082-1.092, 2010.

FERREIRA JUNIOR, I. Institutos de pesquisa do Paraná e o uso de seus recursos no desenvolvimento de relações cooperativas com empresas: um estudo comparativo de casos. Dissertação (Mestrado em Administração) — Setor de Ciências Sociais Aplicadas, Universidade Federal do Paraná, Curitiba, 2006.

; SEGATTO, A.P. Institutos de pesquisa do Paraná e o uso de seus recursos no desenvolvimento de relações cooperativas com empresas. Revista de Gestão USP - Rege, v. 16, n. 2, p. 1-15, 2009. 
FUCK, M.P.; BONACELLI, M.B. A pesquisa pública e a indústria sementeira nos segmentos de sementes de soja e milho híbrido no Brasil. Revista Brasileira de Inovação, v. 6, n. 1, p. 87-121, jan/jun, 2007.

GOUVEA, R.; KASSICIEH, S. Using resources in R\&D policy planning: Brazil, the Amazon and biotechnology. Technological Forecasting and Social Change, v. 72, n. 5, p. 535-547, 2005.

HUANG, C.C. Knowledge sharing and group cohesiveness on performance: an empirical study of technology R\&D teams in Taiwan. Technovation, v. 29, n. 11, 786-797, 2009.

JONHSON, B.; LUNDVALL, B.A. Promovendo sistemas de inovação como resposta à economia do aprendizado crescentemente globalizada. In: LASTRES, H.M.M.; CASSIOLATO, J.E.; ARROIO, A. (Orgs). Conhecimento, sistemas de inovação e desenvolvimento. Rio de Janeiro: Editora UFRJ/Contraponto, 2005.

JUST, R.E.; HUFFMAN, W.E. The economics of universities in a new age of funding options. Research Policy, v. 38, n. 7, p. 1.102-1.116, 2009.

KIM, L.; NELSON, R.R. Prefácio. IN: (Orgs.). Tecnologia, Aprendizado e Inovação: as experiências das economias de industrialização recente. Campinas: Unicamp, 2005.

MINISTÉRIO DA AGRICULTURA, PECUÁRIA E ABASTECIMENTO. Dados Estatísticos. Disponível em: <www.agricultura.gov.br/ > . Acesso em: 25 mar. 2009.

MENDES, N.; SEGATTO-MENDES, A.P. University-industry technological cooperation for energy efficiency: a case study. Brazilian Administration Review, v. 3, n. 1, p. 31-45, jan/jun, 2006.

NEUMAN, W. L. Social research methods: qualitative and quantitative approaches. 3. ed. Boston: Allyn and Bacon. 1997.

NOVELI, M. Cooperações tecnológicas universidade-empresa em parques tecnológicos: estudo de casos múltiplos no Tecnopuc. Dissertação (Mestrado em Administração) - Setor de Ciências Sociais Aplicadas, Universidade Federal do Paraná, Curitiba, 2006.

NUMMELA, N. Looking through a prism: multiple perspectives to commitment to international R\&D collaboration. Journal of High Technology Management Research, v. 14, p. 135-149, 2003.

PARK, H.W.; LEYDESDORFF, L. Longitudinal trends in networks of university-industry-government relations in South Korea: the role of programmatic incentives. Research Policy, v. 39, n. 5, p. 640-649, 2010. 
PEREZ, C. Revoluciones tecnológicas, cambios de paradigma y de marco socioinstitucional. IN: ABOITES, J.; DUTRÉNIT, G. Innovación, aprendizaje y creación de capacidades tecnológicas. México: Universidad Autónoma Metropolitana, Unidade Xochimilco, 2004. p. 13- 46.

PLONSKI, G. A. Cooperação universidade-empresa: um desafio gerencial complexo. Revista de Administração da USP, v. 34, n. 4, p. 5-12, out./dez. 1999.

PORTER, M.E. A vantagem competitiva das nações. Rio de Janeiro: Campus, 1990.

PORTO, G.S. Características do processo decisório na cooperação empresa-universidade. Revista de Administração Contemporânea, v. 8, n. 3, p. 29-52, jul./set. 2004.

et al. Instituto de Pesquisa Tecnológica de São Paulo — IPT: um estudo do perfil de seus relacionamentos. IN: SIMPÓSIO DE GESTÃO DA INOVAÇÃO TECNOLÓGICA, 24. Gramado, RS. Anais... Gramado, 2006.

PRADO, F.O.; PORTO, G.S.; MECENAS, D.S. A gestão da interface instituto de pesquisa/empresa: uma experiência bem-sucedida. In: ASSEMBLEIA DO CONSELHO LATINO-AMERICANO DE ESCOLAS DE ADMINISTRAÇAO — CLADEA, 37, Porto Alegre, RS. Anais... Porto Alegre, 2002.

QUENTAL, C.; GADELHA, C. Incorporação de demandas e gestão de P\&D em institutos de pesquisa. Revista de Administração Pública, v. 34, n. 1, p. 57-78, jan./mar. 2000 .

; FIALHO, B.C. O papel dos institutos públicos de pesquisa na inovação farmacêutica. Revista de Administração Pública, v. 35, n. 5, p. 135-161, 2001.

RAPINI, M.S.; RIGHI, H.M. O Diretório dos Grupos de Pesquisa do CNPq e a interação universidade-empresa no Brasil em 2004. Revista Brasileira de Inovação, v. 5, n. 1, p. 131-156, jan./jun. 2006.

REIS, D.R. Gestão da inovação tecnológica. Barueri: Manole. 2004.

RIBEIRO, M.T.F. A redefinição das agendas dos centros de P\&D: os limites do mercado na sinalização das áreas estratégicas. Revista de Administração Contemporânea, v. 4, n. 2, p. 87-107, maio/ago. 2000.

ROGERS, E. Diffusion of innovations. 5 ed. New York: Free Press, 2003.

SÁEZ, C.B.; MARCO, T.G.; ARRIBAS, E.H. Collaboration in R\&D with universities and research centers: an empirical study of Spanish firms. $R \& D$ Management, v. 32, n. 4, p. 321-341, 2002. 
SANTAMARÍA, L.; BARGE-GIL, A.; MONDREGO, A. Public selection and financing of R\&D cooperative projects: credit versus subsidy funding. Research Policy, v. 39, n. 4, p. 549-563, 2010.

SCHUMPETER, J. Economic theory and entrepreneurial history. Revista Brasileira de Inovação, Rio de Janeiro, v. 1, n. 2, jul./dez. 2002.

SEGATTO, A.P. Análise do processo de cooperação tecnológica universidade-empresa: um estudo exploratório. 175 f. Dissertação (Mestrado em Administração) — Universidade de São Paulo, São Paulo, 1996.

SEGATTO-MENDES, A.P.; ROCHA, K.C. Contribuições da teoria de agência ao estudo dos processos de cooperação tecnológica universidade-empresa. Revista de Administração da USP, v. 40, n. 2, p. 172-183, 2005.

; SBRAGIA, R. O processo de cooperação universidade-empresa em universidades brasileiras. Revista de Administração da USP, v. 37, n. 4, p. 58-71, out./dez. 2002.

SHARIF, N. Emergence and development of the national innovation system concept. Research Policy, v. 35, n. 5, p. 745-766, 2006.

SOUZA, J.G. Cooperação escola-empresa: uma ponte entre educação e desenvolvimento regional. In: SIMPÓSIO DE GESTÃO DA INOVAÇÃO TECNOLÓGICA, 22. Salvador, BA. Anais... Salvador, 2002.

STEINER, J.E. Institutos de pesquisa: missão, liderança e inovação. Revista Parcerias Estratégicas, v.20, n. 5, p. 1.371-1.377, jun. 2005.

SUURS, R.A.A.; HEKKERT, M.P. Cumulative causation in the formation of a technological innovation system: the case of biofuels in the Netherlands. Technological Forecasting and Social Change, v. 76, n. 8, p. 1.003-1.020, 2009.

TEECE, D.J. Profiting from technological innovation - implications for integration, collaboration, licensing and public policy. Research Policy, v. 15, p. 285-305, 1986.

TIGRE, P.B. Gestão da inovação: a economia da tecnologia no Brasil. Rio de Janeiro: Elsevier. 2006.

TUZI, F. Useful science is good science: empirical evidence from the Italian National Research Council. Technovation, v. 25, p. 505-512, 2005.

VEDOVELLO, C. Science park and university-industry interaction: geographical proximity between the agents as a driving force. Technovation, v. 17, n. 9, p. 491502, 1997.

YIN, R. K. Estudo de caso: planejamento e métodos. 3. ed. Porto Alegre: Bookman, 2005. 